\title{
An Analysis of Mangosteen-skin (Garcinia mangostana L.) Extract Gel to Increase the Number of Fibroblast and Collagen Width During Wound Healing Process after Tooth Extraction of Guinea Pigs
}

\author{
${ }^{1}$ Hendri Poernomo, ${ }^{1,2}$ Mochammad Taha Ma'ruf, 'Durra Mufida \\ ${ }^{1}$ Department of Oral and Maxillofacial Surgery, \\ ${ }^{2}$ Department of Biomedical Science, Mahasaraswati University of Denpasar, \\ Denpasar, Bali, Indonesia
}

\begin{abstract}
The objective of this research is to determine the application effect of mangosteen-skin (Garcinia mangostana L.) extract gel to the number of fibroblast and collagen width during wound healing process after tooth extraction of guinea pigs. In vivo experimental study consist of 24 guinea pigs divided into 4 groups. Extraction of lower right incisive performed and mangosteen-skin extract gel applied later with different concentration of 20,40 and $60 \%$ for each group. On the 7 th day, guinea pigs were decapitated and prepared using Haematoxylin Eosin (HE) and Mallory staining. The number of fibroblast cell and collagen matrix width is measured using binocular microscope. Result shows significant difference on the number of fibroblast and collagen width among experimental group ( $\mathrm{p}<0.05$ ). Mangosteen-skin extract gel with $40 \%$ concentration further increased the number of fibroblast and collagen width than the rest of the group of $20 \%$ concentration, $60 \%$ concentration and control group. It could be concluded that mangosteen-skin extract gel with $40 \%$ concentration could accelerate wound-healing process after tooth extraction of guinea pig, indicated by increasing number of fibroblast and collagen width. This condition achieved because of the mangosteen-skin containing gamma-mangosteen an active compound with anti-inflammation activity. Gamma-mangosteen blocked the transformation process of arachidonic acid to $\mathrm{PGE}_{2}$ on the cyclooxygenase track.
\end{abstract}

Key words: Mangosteen-skin extract, Garcinia mangostana L. wound healing, concentration, transformation, concluded

\section{INTRODUCTION}

Tooth extraction was a common procedure in dentistry. Tooth extraction will create an open wound; normally will lead to a complete wound healing without any complication. Nevertheless, it will lead to several complications frequently and will slow down the wound-healing process (Steiner et al., 2008). Wound healing after tooth extraction is very important, especially in case of continuing further treatment such as dentures, implant and braces.

Wound healing is a specific biologic process related to tissue growth and regeneration. This process consists of granulation tissue formation containing inflammation cells, new blood vessel and fibroblast bonded to extracellular collagen matrix (Favia et al., 2007). Wound healing consists of several phases; haemostatic and inflammation phase, proliferation phase and maturation phase. During proliferation phase, fibroblast from undifferentiated mesenchyme cell plays a major role.
Fibroblast will produce collagen fiber based material to link the wound edge. Fibroblast will also create a new connective tissue providing strength and integrity resulting to a good wound-healing process. Enhancement of the number of fibroblast will increase the number of collagen fiber resulting to acceleration of wound healing (Schwartz et al., 2000).

To cure various diseases, the community has widely used medicinal plant. The middle-down community purchasing power on natural based medicine had increased due to the high price of modern medicine. One of the natural ingredients that can be used potentially was a mangosteen (Garcinia mangostana L.) Mangosteen tree component can potentially cure various diseases. Study of Yatman (2013) shows that mangosteen contains many xanton within it skin $(107.76 \mathrm{mg} / 100 \mathrm{~g})$. Xanton is a natural chemical substance in polyphenolic compound that cannot be found in other fruits. According the study (Nugroho, 2008), xanton substance contains active ingredients that are gamma-mangostin with

Corresponding Author: Hendri Poernomo, Department of Oral and Maxillofacial Surgery, Mahasaraswati University of Denpasar, Bali, Denpasar, Indonesia 
anti-inflammation characteristic. Gamma-mangostin blocks cyclooxygenase track and potentially blocks the release of Prostaglandin E2(PGE2), important mediator during inflammation reaction (Nakatani et al., 2002).

Further study by Nakatani et al. (2002), mangosteen-skin extracted by $40 \%$ ethanol more Potentially could block $\operatorname{IgE}$ during histamine release and prostaglandin E2(PGE2) synthesize rather than mangosteen-skin extracted by $70,100 \%$ ethanol and water. Other study of Nadiawati (2013) reveals that mangosteen-skin extract gel with $40 \%$ concentration extracted by $70 \%$ ethanol could accelerate collagen density during gingival wound-healing process. Based on the two studies above in this study mangosteen-skin was extracted using $40 \%$ ethanol and divided into several groups as 20,40 and $60 \%$.

The aim of the study was to determine the comparison of mangosteen-skin extract gel with various concentrations to the enhancement of fibroblast number and collagen width during wound healing after tooth extraction in guinea pigs. From this study we expect to develop knowledge in new substance and potentially as herbal medicine to accelerate wound healing following extraction procedure, by exploitation of wasted mangosteen-skin.

\section{MATERIALS AND METHODS}

Mangosteen-skin extract gel, CMC-Na gel, chloroform, Harris Haematoxylin-Eosin stain, Mallory stain, Guinea pig (Cavia cobaya), electronic scale, homogenizer, Erlenmeyer tube, vacuum rotary evaporator, water bath, filter study, automatic tissue processor, paraffin block cast, freezer, microtome, hot plate, staining jar, object glass, deck glass, binocular microscope (Olympus Type CX31).

Mangosteen-skin extract preparation: Mangosteen-skin extract (Garcinia mangostana L.) was obtained by maceration using $40 \%$ ethanol solution. Mangosteen-skin were cut into small pieces and left inside dryer with temperature $50^{\circ} \mathrm{C}$ for 4 days. The mangosteen-skin then turned into powder using powdering machine and filtered. Ethanol $40 \%$ were added into the dried mangosteen-skin powder, later stirred using electric mixer for $30 \mathrm{~min}$ and leaved for $24 \mathrm{~h}$ before filtered using Buchner cone. The process was repeated 3 times until dregs and filtrates obtained. Stiff extract were vaporized using water bath $70^{\circ} \mathrm{C}$. Final result were poured into porcelain cup and reheated until $70^{\circ} \mathrm{C}$ and mangosteen-skin extract obtained.

Mangosteen-skin extract gel preparation: Mangosteen skin extract $(10,20,30 \mathrm{mg})$ added with $1.00 \mathrm{G}$ CMC-Na gel and $180.00 \mathrm{mg}$ methyl paraben, homogeny stirred, hot aquadest added later until $50 \mathrm{~mL}$ volume obtained and homogeny stirred until 20,40 and $60 \%$ concentration obtained. Control group prepared by dissolving $1.00 \mathrm{G}$ CMC-Na in hot aquadest by $50 \mathrm{~mL}$ homogeny stirred.

In vivo study: The sample of study consist of 24 guinea pigs (male, 3 months old, 250-300 G body weight, healthy). Divided into 4 groups. Every guinea pigs has been anesthetized with ketamine $(0.2 \mathrm{~mL} / \mathrm{kg} \mathrm{BW}$, intra muscular) in the upper thigh. Lower left incisive being luxated using excavator and then extracted using haemostat forceps. Among control group (C)CMC-Na gel topically applied into the socket, Group $1(\mathrm{G} 1)$ topically applied by $20 \%$ mangosteen-skin extract gel, Group 2(G2) topically applied by $40 \%$ mangosteen-skin extract gel and Group 3(G3) topically applied by $60 \%$ mangosteen-skin extract gel. On the 7 th day after tooth extraction, guinea pigs were decapitated. Post-extraction socket and surrounding bone has been cut and cleaned using $\mathrm{NaCl}$ solution and fixated with $10 \%$ buffered formalin for $24 \mathrm{~h}$ to preserve the stability of the surrounding tissue physically and chemically. The histological examination was performed later.

A fibroblast examination was performed using Haematoxylin Eosin staining and collagen examination was performed using Mallory staining. A fibroblast cell counting was obtained from surrounding socket of guinea pigs from the apex towards cervical. Pictures have been taken onto fibroblast cell and collagen matrix and measured using measuring software in micrometer. Fibroblast cell and collagen matrix were examined using binocular microscope (Olympus Type CX31). Counting was performed in 5 field of view; the number of fibroblast was counted later in every field of view. Field of view 1 till 5 was summed and the averaged was taken.

\section{RESULTS AND DISCUSSION}

Number of fibroblast: Analysis of treatment effect was performed based on average number of fibroblast between the groups after treatment. The significance analysis using one way ANOVA was presented in Table 1 (Fig. 1 and 2).

Table 1 reveals that the average number of fibroblast in $40 \%$ mangosteen-skin extract gel group (G2) was the highest among other groups. One way ANOVA test in Table 1 reveals that the average of fibroblast number among the four groups were significantly different $(p<0.05)$ after treatment. Least SignificantDifference (LSD) has been performed to observe difference between (Table 2). 
(a)

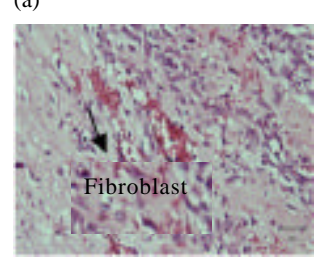

(c)

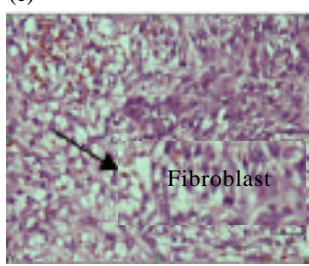

(b)

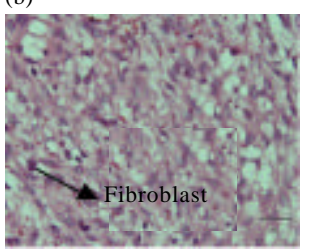

(d)

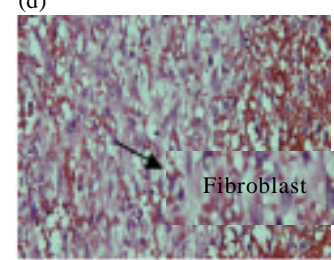

Fig. 1: Fibroblast; a) Control group; b) $20 \%$ concentration c) $40 \%$ concentration and d) $60 \%$ concentration
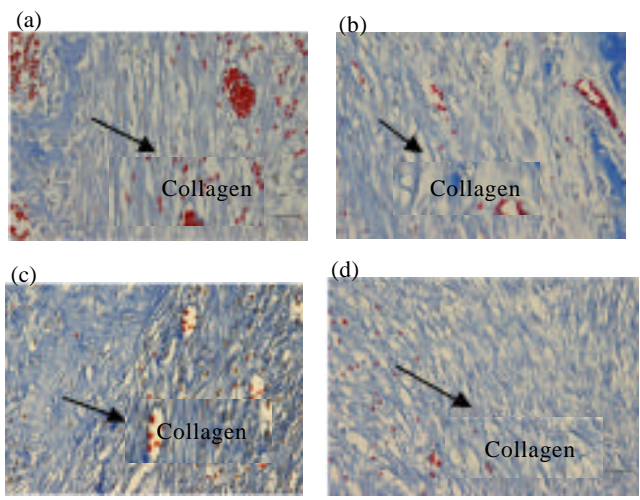

Fig. 2: Collagen; a) Control group; b) $20 \%$ concentrations; c) $40 \%$ concentration and d) $60 \%$ concentration

Collagen width: Analysis of treatment effect was performed based on average of collagen width between the groups after treatment. The significance analysis using one way ANOVA was presented in Table 3. Table 3 reveals that the average of collagen width in $40 \%$ mangosteen-skin extract gel group (G2) was the highest amongst the groups. One way ANOVA test in Table 3 reveals that the average of fibroblast number among the four groups were significantly different $(p<0.05)$ after treatment. Least Significant Difference (LSD) has been performed to observe difference between groups (Table 4).

From the study, during wound-healing process after tooth extraction of guinea pigs, the number of fibroblast were increased among group of $40 \%$ mangosteen-skin extract gel, rather than other groups of 20 and $60 \%$ concentration of mangosteen-skin extract gel. Active compounds of xanton carrying an anti inflammation

Table 1: Difference of averages of fibroblast number between groups $(\mathrm{n}=5)$

\begin{tabular}{lcrc}
\hline Groups & Average of fibroblast & F-value & $p$-value \\
\hline C & $215.17 \pm 24.73$ & 31.80 & 0.001 \\
G1 & $282.86 \pm 24.87$ & & \\
G2 & $347.23 \pm 22.19$ & & \\
G3 & $289.97 \pm 22.02$ & & \\
\hline
\end{tabular}

Table 2: Least significant difference test of fibroblast number between groups

\begin{tabular}{llll}
\multicolumn{1}{c}{$(\mathrm{n}=5)$} & & & \\
\hline Groups & G1 & G2 & G3 \\
C & 0.001 & 0.001 & 0.001 \\
G1 & & 0.001 & $0.606^{*}$ \\
G2 & & & 0.001 \\
\hline *Significant values & & &
\end{tabular}

*Significant values

Table 3: Difference of average of collagen width between groups ( $\mathrm{n}=5$ )

\begin{tabular}{llcr}
\hline Groups & Average of collagen & F-value & p-value \\
\hline C & $554.54 \pm 84.01$ & 59.73 & 0.001 \\
G1 & $829.73 \pm 64.51$ & & \\
G2 & $1097.72 \pm 69.53$ & & \\
G3 & $863.17 \pm 61.94$ & & \\
\hline
\end{tabular}

Table 4: Least significant difference test between groups for collagen width

\begin{tabular}{llll}
\multicolumn{4}{c}{ after application of mangosteen-skin extract gel $(\mathrm{n}=5)$} \\
\hline Groups & G1 & G2 & G3 \\
\hline C & 0.001 & 0.001 & 0.001 \\
G1 & & 0.001 & $0.421^{*}$ \\
G2 & & & 0.001 \\
\hline "Significant value & & &
\end{tabular}

activity were gamma-mangostin. Gamma-mangostin blocks the transformation of arachidonic acid to PGE2 inside the microsom, blocking in cyclooxigenase track. During lack of PGE2 production, vasodilatation of the blood vessel will be suppressed resulting to obstruction of macrophage activity to the injury site (Astawa and Bakta, 2007). Gamma-mangostin potentially could block PGE2 release in rat glioma cell. During in-vitro enzymatic test, this compound was being able to block COX-1 and CX-2 enzyme activity. During inflammation process, gamma-mangostin blocks Ikappa kinase enzyme activity directly, to prevent COX-2 gen transcription process and reduce the production of PGE2 (Nakatani et al., 2002).

Fibroblast cells was the major component during wound-healing process, originated from mesenchyme tissue which also an embryonic tissue for connective tissue, bone tissue and cartilage. Fibroblast cell produce extra-cellular matrix for connective-tissue (Khoswanto, 2010). In this study, fibroblast cell has been used as main indicator during the wound-healing process because proliferation and migration of fibroblast cell into granulation tissue were needed during wound closure, followed by extracellular matrix component deposition, wound contraction and remodelling. In fact, fibroblast cell were capable to support neo-angiogenesis, secretion of extracellular matrix (glikosaminoglikan, proteoglikan, glycoprotein and collagen) and produce some cytokine and growth factor (Mariggio et al., 2009). Increasing number of fibroblast on wound site represents a 
combination of proliferation and migration. These fibroblast were originated from local mesenchymal, especially associated with adventitia layer their growth were caused by cytokines produced by macrophage and lymphocyte (Triyono, 2005).

During wound-healing proliferation-phase, fibroblast cell produce cytokine and growth factor as TGF-•, CTGF, IL-6 and IL-8 was a pleiotropic growth factor synthesize by different cell type in three isoform. During wound healing, platelet and macrophage have a role as a trigger to the formation of TGF-• . TGF-• stimulates migration and proliferation of fibroblast cells, supporting metalloproteinase's matrix to produce collagen and increase angiogenesis with Vascular Endothelial Growth Factor (VEGF). Furthermore, TGF-• stimulates reepithelialisation and fibroblast transformation into myofibroblast (Mariggio et al., 2009). Macrophage wills later develope granulation tissue which will attract fibroblast as the biggest number component in granulation tissue to the injury site and starting the collagen synthesize (Nadiawati, 2013).

Collagen is the most numerous protein found among human and animal. The role of collagen associated with the formation of new collagen fiber by fibroblast to help tissue restoration process. Collagen synthesize begins with the formation of procollagen, transported to endoplasm reticulum and forming triple helix a stronger bond with other two procollagen. Formed procollagen bond exit the cell, later enzyme will split the end of procollagen, so that, this small fragment will configure collagen. Later, collagen molecule will configure fibril collagen and collagen fibre established (Mawardi et al., 2002).

In this study, the increasing number of fibroblast along with an increasing of collagen width also allegedly by the presence of pharmacologic effect of active compound ingredient from mangosteen-skin extracted with ethanol, beside xanton. Mangosteen-skin extract contains chemical component of alkaloid, saponin, flavonoid, tannin, steroid or triterpenoid and glycosides. Saponin could trigger Vascular Endothelial Growth Factor (VEGF) and increase the number of macrophage migrated to injury site with the result to increase cytokine production which will activate fibroblast on injury site (Poeloengan and Praptiwi, 2010; Kimura et al., 2006).

Previous study on some plants reveals that flavonoid hold anti-oxidant, anti-microbe and anti-inflammation activity. Anti-inflammation activity caused by the bensopiron chain in the flavonoid structure were able to bond with cyclooxygenase and lipooxygenase enzyme. Moreover, flavonoid contains hydroxyl cluster on C5 and C7 that bonded to lipooxygenase enzyme. Flavonoid is able to block the transformation of arachidonic acid into PGE2 and leukotrien by the blocking of cyclooxygenase and lipooxygenase track. Blocking of leukotrien production could take effect to the number of inflammation cell because of the major role of leukotrien in chemo tactic during inflammation (Narayana et al., 2001).

Tannin was a water-soluble phenol compound. Contents of tannin are useful as an astringent or stops bleeding, accelerate wound healing and mucosal membrane inflammation and new tissue regeneration (Reddy et al., 2012). Triterpenoid could also support the wound healing process with the same activity like tannin as an astringent and antimicrobial. Steroid and phenol compound within mangosteen-skin plays a role in wound healing including as an anti oxidant destroying free radicals, decreasing lipid oxidation, relieving necrotic cell and increase vascularization (Soni and Singhai, 2012).

\section{CONCLUSION}

It could be concluded that mangosteen-skin extract gel with $40 \%$ concentration could accelerate wound-healing process after tooth extraction of guinea pig, indicated by an increased number of fibroblast and collagen width. This condition achieved because mangosteen-skin contains gamma-mangosteen an active compound with anti-inflammation activity. Gamma-mangosteen blocked the transformation process of arachidonic acid to PGE2 on the cyclooxygenase track.

\section{REFERENCES}

Astawa, P. and M. Bakta, 2007. [Makrofag expression il-1? As well as the systemic inflammatory response to early internal fixation of the closed femoral fracture is lower than that delayed (In Indonesia)]. Indonesian $\mathrm{J}$. Biomed. Sci., 1: 1-12.

Favia, G., M.A. Mariggio, F. Maiorano, A. Cassano and S. Capodiferro et al., 2007. Accelerated wound healing of oral soft tissues and angiogenic effect induced by a pool of aminoacids combined to sodium hyaluronate (AMINOGAM). J. Biol. Regulators Homeostatic Agents, 22: 109-116.

Khoswanto, C., 2010. The effect of mengkudu gel (Orinda citrifolia Linn.) in accelerating the ecalation of fibroblast post extraction. Dent. J., 43: 31-34.

Kimura, Y., M. Sumiyoshi, K. Kawahira and M. Sakanaka, 2006. Effects of ginseng saponins isolated from Red Ginseng roots on burn wound healing in mice. Br. J. Pharm., 148: 860-870. 
Mariggio, M.A., A. Cassano, A. Vinella, A. Vincenti and R. Fumarulo et al., 2009. Enhancement of fibroblast proliferation, collagen biosynthesis and production of growth factors as a result of combining sodium hyaluronate and aminoacids. Intl. J. Immunopathol. Pharmacol., 22: 485-492.

Mawardi, H., L. Dalimi and S. Darmo, 2002. The effect of local propolis extract application on the process of formation of collagen fibers post-extraction of marmot tooth (Cavia cobaya). Health Sci., Vol. 15,

Nadiawati, R.P., 2013. [Effect of gel application of mangosteen skin extract (Garcinia mangostana $\mathrm{L}$.) on collagen fiber density on gingival wound healing process (Study on Rattus Norvegicus)]. Ph.D Thesis, Gadjah Mada University, Yogyakarta, Indonesia. (In Indonesia).

Nakatani, K., N. Nakahata, T. Arakawa, H. Yasuda and Y. Ohizumi, 2002. Inhibition of cyclooxygenase and prostaglandin E 2 synthesis by ?-mangostin, a xanthone derivative in mangosteen, in C6 rat glioma cells. Biochem. Pharmacol., 63: 73-79.

Narayana, K.R., M.S. Reddy, M.R. Chaluvadi and D.R. Krishna, 2001. Bioflavonoids classification, pharmacological, biochemical effects and therapeutic potential. Indian J. Pharmacol., 33: 2-16.

Nugroho, A.E., 2008. [Mangosteen (Garcinia mangostana L.): From wasted fruit leather to become candidate a drug]. Ph.D Thesis, Universitas Gadjah Mada, Yogyakarta, Indonesia. (In Indonesia).
Poeloengan, M. and P. Praptiwi, 2010. [TEST of antibacterial activity of mangosteen peel skin extract (Garcinia mangostana Linn) (In Indonesia)]. Media Res. Health Dev., 20: 65-69.

Reddy, G.A.K., B. Priyanka, C.S. Saranya and C.K.A. Kumar, 2012. Wound healing potential of Indian medicinal. Int J. Pharm Rev. Res., 2: 79-79.

Schwartz, S.I., G.T. Shires and F.C. Spencer, 2000. [The Essence of Surgical Principles]. 6th Edn., EGC Medical Book Store, Jakarta, Indonesia, (In Indonesia).

Soni, H. and A.K. Singhai, 2012. A recent update of botanical for wound healing activity. Intl. Res. J. Pharm., 3: 1-7.

Steiner, G.G., W. Francis, R. Bureell, M.P. Kallet and D.M. Steiner et al., 2008. The healing socket and socket regeneration. Compend Contin. Educ. Dent., 29: 114-116.

Triyono, B., 2005. [The difference in the appearance of collagen around the incision wounds in wistar mice given infiltration of levobupivacaine pain relievers and those not given levobupivacaine]. Ph.D Thesis, Diponegoro University, Semarang, Indonesia. (In Indonesia).

Yatman, E., 2013. [Mangosteen's skin contains high efficacious xantons (In Indonesia)]. Widya Sci. Mag., 29: $2-9$. 